\title{
Winds from Cataclysmic Variables
}

\author{
J. E. Drew
}

Physics Department, Imperial College of Science, Technology $\mathcal{E}$ Medicine, Blackett Laboratory, London, SW7 2BZ, England

\begin{abstract}
The winds associated with high states of non-magnetic (diskaccreting) cataclysmic variables are described and discussed. A quick summary of the basic phenomenology is given, and followed by a presentation of some of the more important recent developments in our understanding. The near-ubiquity of orbital-phase linked variability of the UV resonance lines (generally thought of as mainly wind-produced) is noted and its implications are considered. The impact of the much lower-thanexpected boundary layer luminosity upon mass loss rate determinations is also discussed. Current work on the role of radiation pressure (mediated by line opacity) is placed in context.
\end{abstract}

\section{Introduction}

This is a review of our knowledge of one particular class of disk wind - the mass loss associated with "non-magnetic" cataclysmic variables.

After describing the basic phenomenology and our understanding of it, the focus will move onto the developments of the past decade (some welcome, one less so!). An assessment is then made of how well we are doing at estimating mass loss rates for these systems. Finally the scene is set for some much needed work on the problem of the dynamical origin of CV winds. No one can yet claim that the agent (or agents) responsible for driving these outflows has been positively identified. This provides a contrast to the consensus that has developed among those working on young stellar objects that magnetic fields play a fundamental role. Magnetic fields may also be important in shaping CV winds, but since the best-developed outflows are associated with the luminous discs of CV where the stellar magnetic field is believed to be relatively weak, it remains possible that radiation pressure is significant. At the time of writing, two groups are independently exploring models of radiation-driven disk winds for $\mathrm{CV}$ after a period of years in which the concept has lain fallow. In view of this, it is a good moment to look at the background to this problem.

\section{Phenomenology}

Clear unambiguous spectroscopic signatures of mass loss are to be found only in the outburst state of the so-called non-magnetic CV. These are systems in which a luminous, optically-thick disk, in contact with the white dwarf surface, is believed to be present. 
At optical wavelengths the signature of mass loss is typically subtle. As is well-known, the optical spectra of high-state CV exhibit strong Balmer and helium line absorption or emission (depending somewhat on the disc's orientation with respect to the observer) that can, in the main, be attributed to the accretion disk. However, it has been noted that the line emission seen in certain eclipsing nova-like variables is not double-peaked. This does not fit easily with ideas on line formation in a Keplerian disc, and has been viewed as evidence of an outflow component in the affected systems (see Robinson et al. 1993). Extra force to this interpretation has recently come from calculations by Murray \& Chiang (1996) in which it is demonstrated that the modest velocity shear linked with the onset of outflow away from the disk plane could well be sufficient to bring about this effect (see also Horne 1995).

At ultraviolet wavelengths the relativities are reversed in that the prominent heavy-element resonance lines bear the clear mark of mass loss in the form of a blueshifted, broad absorption component. This, at least, is the case for noneclipsing systems where the optically-thick disk is shadowed by the resonantly scattering ions moving with the flow away from the disk plane towards the observer. With a more nearly edge-on disk in systems exhibiting continuum eclipses, broad blueshifted absorption is supplanted by almost as broad, weaklyeclipsing, roughly symmetric line emission. This is easily explained by the bulk of the wind no longer being 'back-lit' by the continuum-emitting disk (what was absorption at low inclination due to scattering out of the line of sight, is now emission due to scattering back into it).

The best developed of the ultraviolet line profiles is usually C IV $\lambda 1549$. $\mathrm{N}$ v $\lambda 1240$ and $\mathrm{Si}$ IV $\lambda 1397$ are often strong also. The velocity broadening of these lines in mass-losing CV is usually in the range $3000-6000 \mathrm{~km} \mathrm{~s}^{-1}$. Such speeds and the small size of these binaries $\left(10^{10} \lesssim a \lesssim 10^{11} \mathrm{~cm}\right)$ imply very short flow timescales on the order of 100 secs or so. It was quickly recognised that the high outflow speeds observed were likely to mean that $\mathrm{CV}$ winds originate from close to, if not on, the accreting white dwarf (Cordova \& Mason 1982). This is because it is commonly the case that the maximum speeds attained in a wind are comparable with the footpoint escape speed.

A further important observed property of the mass loss signatures in nonmagnetic $\mathrm{CV}$ is that they appear, in the ultraviolet at least, to be very sensitive to luminosity. Several instances of this might be quoted. Declines of dwarf novae from maximum light have often been caught by the International Ultraviolet Explorer satellite. As the ultraviolet continuum decays by a factor of two or a few, it is commonplace to see the blueshifted absorption prominent at maximum light all but vanish (e.g. Woods et al. 1992). Analogous behaviour has been noted in nova-like variables as their brightness level varies (Figure 1 in Mason et al. 1995 affords a striking example of this). It is probably symptomatic of the same dependence that the wind effect is typically weak in some high-state systems and very marked in others (this is well-demonstrated by the IUE high spectral resolution data collected together by Prinja \& Rosen 1995).

At first, the discovery of weak or no ultraviolet line eclipses in systems presenting deep continuum eclipses was taken to imply extension of the line-forming volume out to radii comparable with the companion star radius. It was shown by Drew (1987) that this conclusion can be avoided if the out-of-eclipse line profile 
is, in effect, a spatially unresolved superposition of a net absorption component on a stronger emission feature - which it very plausibly may be in these systems. At the same time it was also shown that the non-detection of blueshifted absorption in the UV spectra of high-inclination systems was evidence that the outflow must be somewhat bipolar and that gradual acceleration of the outflow offered the best prospect of fitting the absorption profiles seen in non-eclipsing CV (Drew 1987, Mauche \& Raymond 1987).

The 1980s saw a number of attempts to make sense of the degree of ionization apparent in CV winds (e.g. Kallman \& Jensen 1985, Drew \& Verbunt 1985 , Mauche \& Raymond 1987). All were dogged by the problem of the fiercely ionizing effect of the radiation thought to be produced in the boundary layer between the inner edge of the accretion disk and the white dwarf. The radiation of half the accretion luminosity (as required for the case of white dwarf rotating at a modest fraction of break-up) by a surface unlikely to be much greater than that of the entire white dwarf is certain to be very 'hot'. In as complete a calculation as has ever been attempted, Mauche \& Raymond (1987) showed that the presence of a full-power boundary layer, in addition to a UV bright accretion disk, could only be reconciled with the observed C IV and similar features if the mass loss rate is comparable to the mass accretion rate. There is no dynamical theory in a state, yet, to suggest that this is too high to be sustained. However, if the mass loss rates really are so high, radiation pressure is damaged as a contender for the driving mechanism since the single-scattering momentum limit lies at $\dot{M} \sim 0.01 \dot{M}_{\text {acc }}$.

\section{Developments of the past decade}

\subsection{The boundary layer}

In 1987, particularly after the publication of the study by Mauche \& Raymond, it looked as though a key problem to tackle was the seemingly high mass loss rate required to counterbalance the ionizing effect of a luminous boundary layer. Accordingly it has been a relief that evidence has since appeared that the boundary layers in CV do not commonly radiate anything like half of the accretion luminosity.

Direct estimations of the ratio between the boundary luminosity and that released by the disk have been derived from Voyager and EXOSAT observations of VW Hydri in outburst (Mauche et al. 1991) and more recently for SS Cygni in outburst based on EUVE data (Mauche et al. 1995). In both cases the energy budget apportions no more than 10 percent of the maximum achievable luminosity to the boundary layer. Hoare \& Drew (1991) reached a similar conclusion, indirectly, for a larger sample of CV on applying the Zanstra method to the HeII $\lambda 1640$ and $\lambda 4686$ lines - the idea here was to use the probable wind contribution to the He II emission to set limits on the $\mathrm{He}^{+}$ionizing continuum "seen" by the wind which would have to be dominated by the boundary layer contribution.

As ROSAT data has appeared (Van Teeseling et al., 1994, 1995) the general picture of a relatively feeble boundary layer component has been confirmed. Furthermore, in one or two high-inclination systems, the failure to detect clear X-ray eclipses at times of optical eclipse may indicate that not all the X-ray light comes from a white-dwarf sized or smaller object (e.g. Wood et al. 1995). 
The first soft X-ray/extreme UV spectral observations that are now emerging from EUVE are showing these energies present a bewildering complex of line features that, as yet, defy quantitative modelling. The bottom line appears to be that $\mathrm{CV}$ boundary layers are typically minority contributors to the total binary luminosity and have a spectral character closer to the optically-thin limit than to that of an optically-thick atmosphere.

This reduction in the effective ionizing flux bathing the disk wind in a $\mathrm{CV}$ certainly feeds through to a reduced mass loss requirement. However there is not yet a convergence of opinion on what the 'saving' is (see section 4.).

\subsection{Variability}

To balance the good news of low-luminosity boundary layers, there has been the appearance of a quite different mystery that has not yet been fully assimilated. IUE observations taken in 1987 (Drew \& Verbunt 1988) of the short-period dwarf nova, YZ Cnc, during one of its outbursts provided the first evidence of strong orbital-phase linked UV resonance line variability in a 'normal' CV (the peculiar, long-recurrence time system WZ Sge had earlier been reported to show possibly regular changes also, ref). The changes in resonance line profile shape were very dramatic - within a quarter of the orbit, broad blueshifted absorption features were seen to increase from almost absent to almost black.

To begin with it looked as though this variability might be a property of just the short-period non-eclipsing SU UMa systems. Certainly the first few systems to show orbital-phase linked changes as marked as in YZ Cnc were also of this type (Woods et al. 1990, 1992). However the phenomenon is not quite so limited. Evidence has accumulated that longer period systems can exhibit line profile variability on the same period as the binary orbit as well. The difference is that the variability is much more subtle, showing up in IUE data as little more than radial velocity changes in the blue shifted absorption (e.g. Mauche 1991, Knigge et al. 1994). Finally, and more recently, it has become apparent that the UV emission features seen in eclipsing systems vary in strength as a function of orbital phase - the most telling data in this regard, by virtue of their high spectral resolution, are the HST observations of UX UMa (Mason et al. 1995, see also the narrow-band light curve approach of Baptista et al. 1995). In short, none of the high-state systems in which winds are present is free of orbital phase-linked variability.

But does the variability have anything to do with the mass loss? The best provisional answer to this is "no". The reasoning is simply that it is unlikely that the mass loss has any memory of the orbital period if it emerges primarily from the inner disk. In addition, a Coriolis effect can be recognised as implausible when it is remembered how very short the flow timescale is compared with the orbital timescale (cf. $\sim 100 \mathrm{sec}$ with 100 minutes or more). The most likely candidate for the blame here must be interaction between the mass transfer stream and the accretion disk as the latter overflows the disk edge and settles. This ensures the appropriate period and offers the prospect of an emissive region whose visibility can be phase-dependent. It also provides a natural explanation for the stronger apparent effect in short-period systems with smaller disks (many of these points were discussed in Knigge et al. 1994). Grasped in these terms, the basic picture of what is going on in these systems is that of an orbital-phase- 
dependent source of UV resonance line emission superimposed on wind-formed line profiles that are steady or, at least, only varying secularly.

We now have a clear warning that an automatic presumption UV line profiles can be modelled as pure wind features may lead us into serious error. There always had to be some concern, particularly where the wind signature is weak, that these profiles include at least some disk photospheric or chromospheric component. For the moment we have been saddled with something potentially even more troublesome - a variable component that may be difficult to characterise fully. In view of these concerns, longer period systems showing the weakest orbital-phase related variability must be the preferred objects of study for those wanting to understand wind phenomena.

At optical wavelengths, considerable attention has been paid to the so-called SW Sex phenomenon. A key aspect of this is the temporary appearance in eclipsing nova-like variables of an absorption component at about orbital phase 0.5 splitting the otherwise single-peaked Balmer and He I emission lines. It is an attractive (Occam's Razor) simplification to take the view that this might be the product of the same property of these systems as the UV orbital-phaselinked resonance line variability. Indeed Hellier \& Robinson (1994) have raised the possibility that stream overflow is responsible for SW Sex behaviour.

\subsection{Technical developments: from IUE to HST, and from simplified central wind models to full disk winds}

The primary advantage of HST as a facility for UV observation of point sources is clearly light grasp. The larger aperture opens up the possibilities of observing at higher time and spectral resolution. Even in outburst, most CV are faint enough that most IUE data have had to be obtained at low dispersion. This capability got us to the position summarised in section 2 . and has provided the database revealing the near-ubiquity of orbital-phase dependent variability.

A flavour for what HST can do for the study of mass loss from CV is to be found in the GHRS study of the nova-like variable UX UMa. This programme yielded high time- and high spectral resolution observations of the C IV 1549 profile (see Mason et al. 1995). The spectral resolution straightaway revealed centrally-located absorption features that are the cause of the non-eclipsing of the emission lines first detected in IUE data (King et al. 1983).

In principle, high time resolution observations obtained during primary eclipses of high inclination systems can be used to better define the geometry and kinematics of the mass loss, as expressed via the resonance line emission, just as continuum eclipse light curves have been used for a decade or so to map the continuum brightness temperature as a function of disk radius. There is a difference however - all three spatial dimensions are involved in the formation of UV (and perhaps some optical!) lines - not just two as in conventional disk eclipse mapping. Given that the 'multi-colour' time series obtained is of the same effective dimensionality as used for continuum mapping, the missing dimension must be made up with model assumptions (i.e. there can be no model-independent reconstruction of the data).

Presently, two collaborations have assembled the tools necessary for theoretical simulation of the eclipse behaviour of UV resonance lines due to a disk wind (see Shlosman \& Vitello 1993, Knigge et al. 1995, Shlosman et al. 1996, 
Knigge \& Drew 1996). A by-product of this spate of activity has been a wider appreciation of the possible significance of the property, first noted by Naylor et al. (1988) in data on OY Car, of narrowing of the UV resonance lines at mideclipse. It is a strong hint that rotational motion is kinematically significant in the line forming region. This has been seized upon as direct evidence that the footpoints of the wind streamlines are located in the disk. Whilst this is an appealing interpretation, underpinned by the results of simulation, it should be recognised that it is not the only one since the fact of the narrowing does not tell us whether the high velocity (rotating) medium occulted at mid-eclipse is located in or above the plane of the disk. Where it can be confirmed that the wind emission in such systems is optically-thick and covers any disk chromosphere then the narrowing really is evidence of a rotating disk wind.

Elsewhere in this volume, Knigge etal. report on comparisons between the UX UMa HST time series observations and Monte Carlo simulations. These suggest the presence of a substantial 'stall' zone above (and below) the disk plane - i.e. that a substantial column of 'turbulent' material is sandwiched between the disk plane and a more diffuse rapidly-accelerated wind. It is also noteworthy that pure scattering had to be supplemented by a contribution from collisional excitation in order to bring the model fluxes into agreement with observation. At this stage the extent to which this contribution accounts for 'contamination' of the line profile by the variable component discussed in section 3.2. is unclear. The fact that the observed $\mathrm{C}$ IV profiles are composites of a wind and a variable component cautions against too literal an acceptance of fits of the data to a pure wind model - the parameters derived from such an exercise should be seen as indicative rather than precise.

\section{On mass loss rate determinations}

At some level, a determination of mass loss rate from wind-formed line profiles involves both profile fitting and an ionization calculation that allows corrections to be made for ion fractions less than unity.

Profile fitting alone provides an estimate of $\dot{M} q$ where $q$ is the ion fraction (the sum of which for a given element must be unity). Accordingly this quantity is very much a lower limit on $\dot{M}$. Prinja \& Rosen (1995) used profile fitting to estimate $\dot{M} q$ from the C IV $\lambda 1549$ line for the sample of objects for which they had collected IUE archive high resolution data (the best data being of bright non-eclipsing systems). They obtained estimates spanning the range $10^{-12}$ to $10^{-11} \mathrm{M} \odot \mathrm{yr}^{-1}$. The rather higher figure of $z 10^{-10} \mathrm{M} \odot \mathrm{yr}^{-1}$ was obtained by Drew (1987) for the eclipsing systems, UX UMa and RW Tri. This last measure gave roughly equal weight to profile fitting and to matching observed line eclipse characteristics. The spread of two orders of magnitude between the low- and the high-inclination systems may be exaggerated. For example, if the line profiles are contaminated by a primarily emissive source (such as that which might be responsible for the orbital-phase linked variability), its effect might be to lower $\dot{M} q$ estimates in non-eclipsing systems where the depth of blueshifted absorption is a key diagnostic, and to raise them in eclipsing systems where a dominant consideration is the matching of the total observed emission line flux. 
To eliminate $q$ from the $\dot{M} q$ product, recourse to an ionization model must be made. For a CV wind, such calculations are both difficult and bedevilled by some awkwards unknowns. The difficult part is that, strictly, almost no approximations can be justified - at the outset, at least. The inner wind is certain to be very dense which means that excited state ionization routes ought not to be ignored (cf. the OB star wind case; Pauldrach 1987, Drew 1989). Radiation transport in the continuum should certainly be treated as there is good reason to suppose that these flows are optically thick in the $\mathrm{He}^{+}$Lyman continuum - and of course this treatment ought to take some account of the twodimensional character of the illuminating radiation field. Furthermore the short flow timescale means that time-independent statistical equilibrium may break down not so far along a given flow streamline - although how far depends on the mass loss rate and on how rapidly the outflow is accelerated up to terminal velocity (Drew \& Verbunt 1985 provide some specific examples).

As described above (section 2.), at the time that Mauche \& Raymond (1987) produced their ionization models, a major unknown was the nature of the boundary layer's emitted radiation. All ionization calculations have had to make assumptions about the outflow geometry and kinematics - given that there has been no firm dynamical model on which they might be hung. Mauche \& Raymond used the simplification of radial outflow. It is hard to tell whether this is such a bad approximation even if, as is now the preferred view, the wind really is a disk wind. Certainly the neglect of rotational motion in an ionization model does not begin to be an issue if the calculation does not also treat excited state ionization processes (so far only Hoare \& Drew 1993 have included any). Only if it becomes clear that most of the mass is not lost from the inner disk the seat of most of the ionizing radiation - should the radial outflow model be condemned outright as too poor an approximation.

In their series of papers, Shlosman \& Vitello set great store on the importance of moving away from one-dimensional treatments (Shlosman \& Vitello 1993; Vitello \& Shlosman 1993; Shlosman et al. 1996). However the quantitative effect of "taking into account the full three-dimensional radiation field" is unclear without appropriate control comparisons. Whilst their treatment of the dilution of the disk radiation field may be accurate, their models apparently do not include a treatment of continuum optical depth or of excited state processes, and the outflow geometry they posit is quite different from that considered in any other published calculations. Despite the benefit of being able to adopt a low-luminosity boundary layer, their models typically return wind mass loss rates in excess of $0.1 \dot{M}_{\text {acc }}$, just a factor of a few down on the estimates of Mauche \& Raymond (1987). This is disappointing.

By contrast, a lower $\dot{M} / \dot{M}_{\text {acc }}$ of $\lesssim 0.05$ was achieved in the models of Hoare \& Drew (1993) for plausible mass accretion rates. In terms of geometry and input physics, these calculations are similar to the Mauche \& Raymond (1987) calculations, the primary differences being a reduced or no role for the boundary layer radiation, and the inclusion of heavy element excited state photoionization routes. Without the latter, the derived mass loss rates would have been (inappropriately) lower still. If indeed CV mass loss rates are in the region of $\lesssim 0.05 \dot{M}_{\text {acc }}$, i.e. comparable with the single-scattering limit, it is easier to imagine that radiation pressure is dynamically significant. 


\section{Wind dynamics and the potential role for radiation pressure}

The one-dimensional radiation-driven wind model as applied to mass loss from OB stars has been a spectacular success. The concept is simple. Momentum is extracted from the radiation field by means of scattering in an enormous number of line transitions. Because the total opacity presented by an incompletely ionized gas in its bound-bound transitions can be $\sim 1000$ times greater than that due to electron scattering opacity alone, the line force can exceed gravity even for stellar luminosities as little as $\sim 10^{-3}$ times the Eddington limit (see Gayley 1996). This, ultimately, is the reason for the ubiquity of mass loss in the upper HR diagram.

Can this success be repeated for the geometry of the accretion disk wind? This question has not yet been answered fully. There are arguments for and against. In the $\mathrm{CV}$ context, it is a good start that high radiative fluxes are produced especially in the inner parts of high-state accretion disks ( $T_{\text {eff }} \gtrsim 50000$ K). Indeed, from consideration of the $T_{\text {eff }} \propto R^{-3 / 4}$ surface brightness distribution, one can see that radiation pressure $\left(\propto R^{-3}\right)$ will do better against gravity $\left(\propto R^{-2}\right)$, nearer rather than further from the accreting white dwarf. In one sense, the fact that an accretion disk wind must be initiated from a reservoir in Keplerian orbit around the accreting star should be an advantage - after all, for trajectories lying in the plane of the disk directed away from the gravity centre, test particles would feel no inward force at all because of the centrifugal balance.

The trouble begins with the fact that radiation pressure vectors tend to be directed upward from the surface of a classical steady state disk (assuming a negligible radial radiation field from a central accreting object). For trajectories out of the disk plane there is a weakening of the centrifugal balance that amounts to an upward increase in effective gravity. This situation is quite different from the one-dimensional stellar wind case where both gravity and radiation pressure decline with increasing radius. The formal implications of this difference were appreciated and discussed by Vitello \& Shlosman (1988) in a semi-analytic study aiming mainly to develop a radiation-driven wind model for AGN disks. They showed, for the expected case of close to vertical streamlines near the disk surface, that a steady-state wind solution is only possible if the line force is an increasing function of velocity in the vicinity of the hydrodynamic critical point - in effect the line force is required to increase with height as does the effective gravity. They mentioned a number of ways in which the natural tendency of the line force to dilute with the radiation field might be reversed to overcome this problem. Their own preference was to appeal to ionization changes. While this option may be appropriate for $\mathrm{AGN}$, it is less plausible for $\mathrm{CV}$.

At the present time, two groups are revisiting this problem within the CV context and are bringing state-of-the-art numerical simulation to bear upon it (see Kallman et al. and Proga et al., these proceedings). Interestingly the conclusions are different, the contrast springing from different perceptions of the way in which the line force varies with height above the disk plane. Kallman et al. have focused on the outer disk and find stable solutions which, ultimately, are admitted by the fact that their formalism suggests the line driving includes a $(z / R)^{\alpha}$ term, where $z$ is height above the disk plane and $\alpha$ is an index with a minimum value of 0.5 . Proga et al. are performing simulations of a radiation 
driven wind from the inner disc. They calculate the line force using a corrected form of a general integral first published by Icke (1980) which, at best, gives a much more modest rise of the line force with height. Hence they find, instead, unsteady wind solutions. These are characterised by a turbulent, clumpy layer close to the disk plane which provides a kind of platform that radiation pressure can act on and strip out to produce a fast, more diffuse outflow. A 'two-stage' wind model such as this is not the first in astronomy: the front-running model for extreme red giant winds is one in which pulsation first levitates material which is then accelerated away by the action of radiation pressure on newly-formed dust (e.g. Bowen \& Willson 1991).

There is a parallel between the time-unsteady wind model and that demanded by the models of the HST observations of UX UMa's C IV $\lambda 1549$ profile mentioned in section 3.3. (Knigge et al. this volume) - the turbulent platform might provide the high column needed to produce the nearly central absorption dips superimposed on the broader emission feature. Both treatments of the radiation driven disk wind problem agree in predicting markedly bipolar (if not quite jet-like) outflow.

Work continues to be in progress in both groups pursuing this problem. An end point has not been reached on how efficient the mass loss might be although it is clear that $\dot{M}$ will show a strong dependence on disk luminosity. There are also subtleties of the radiation transfer to be attended to that may have a big impact on the wind configuration. A further factor to consider is the impact of an added spherically-symmetric component to the radiation field due to the central accreting object - where such a component dominates (e.g. as in the models for AGN recently proposed by Murray et al. 1995) it is clear that a steady-state flow, along streamlines swept back close to the disk plane, is readily achieved. After a thorough exploration of the plausibility of a significant role for radiation pressure in CV winds, it will emerge whether there is a need to exploit channeling by an ordered large-scale magnetic field as well or instead. There should soon be some answers to some interesting questions.

Acknowledgments. JED thanks the IAU for the travel grant that made attendance at Colloquium No. 163 possible. Discussions with Daniel Proga during the preparation of this review are also acknowledged.

\section{References}

Baptista R., et al. 1995, ApJ, 448, 395

Bowen G. H., Willson L. A., 1991, ApJ, 375, L53

Córdova F. A., Mason K. O. 1982, ApJ, 260, 716

Drew J. E., Verbunt F. 1985, MNRAS, 213, 191

Drew J. E., 1987, MNRAS, 224, 595

Drew J. E., 1989, ApJS, 71, 267

Drew J. E., Verbunt F. 1988, MNRAS, 234, 341

Hoare M. G., Drew J. E. 1991, MNRAS, 249, 452

Hoare M. G., Drew J. E. 1993, MNRAS, 260, 647

Horne K. D., 1995, A\&A, 297, 273 
Gayley K. G. 1996, ApJ, 454, 410

Hellier C., Robinson E. L., 1994, ApJ, 431, L107

Icke V., 1980, AJ, 85, 329

Kallman T. R., Jensen K. A., 1985, ApJ, 299, 277

King A. R., Frank J., Jameson R. F., Sherrington M. R., 1983, MNRAS, 203, 677

Knigge C., Drew J. E., Hoare M. G., LaDous C., 1994, MNRAS, 269, 891

Knigge C., Drew J. E. 1996, MNRAS, 281, 1352

Knigge C., Woods J. A., Drew J. E., 1995, MNRAS, 273, 225

Mason K. O., et al. 1995, MNRAS, 274, 271

Mauche C. W., 1991, ApJ, 373, 624

Mauche C. W., Raymond J. C., 1987, ApJ, 323, 690

Mauche C. W., et al., 1991, ApJ, 372, 659

Murray N., Chiang J., 1996, Nature, in press

Murray N., Chiang J., Grossman S. A., Voit G. M., 1995, ApJ, 451, 498

Naylor T., et al. 1988, MNRAS, 231, 237

Pauldrach A., 1987, A\&A, 183, 295

Prinja R. K., Rosen S. R. 1995, MNRAS, 273, 461

Robinson E. L., Marsh T. R., Smak J. I., 1993, Accretion disks in compact stellar systems, ed. J. C. Wheeler, World Scientific, Singapore, p75.

Shlosman I., Vitello P. A., Mauche C. W. 1996, ApJ, 461, 377

Van Teeseling A., Verbunt F., 1994, A\&A, 292, 519

Van Teeseling A., et al., 1995, A\&A, 300, 808

Vitello P. A., Shlosman I. 1988, ApJ, 327, 680

Vitello P. A., Shlosman I. 1993, ApJ, 410, 815

Wood J. H., Naylor T., Marsh T. R., 1995, MNRAS, 274, 31

Woods J. A., Drew J. E., Verbunt F., 1990, MNRAS, 245, 323

Woods J. A., et al., 1992, MNRAS, 255, 237

\section{Discussion}

S. Balbus: What kind of equation of state was used in Proga et al.'s simulation? And what is the origin of the condensates?

J. Drew: The outflow is treated as isothermal, and the origin of the churning motions and condensates is effectively mechanical (this can be understood from the ballistic behaviour of test particles launched vertically from the disk surface at speeds too low to escape the system).

$U$. Torkelsson: Is a radiation-driven wind sufficiently collimated?

J. Drew: At the present time, it may well be for the CV case. 